\title{
Decreased Concomitant Drug AUC
}

National Cancer Institute

\section{Source}

National Cancer Institute. Decreased Concomitant Drug AUC. NCI Thesaurus. Code C54614.

Decreased area under the plot of plasma concentration of a concomitant drug against time after its administration. 